\title{
Archives of Plastic Surgery in the current Warring States period of plastic surgery journals
}

\author{
Seung Yong Song
}

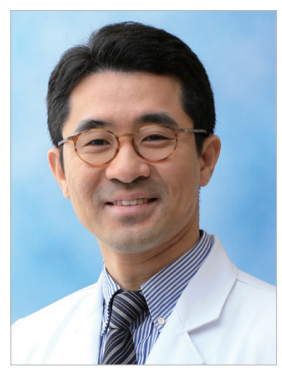

Of late, I have frequently received e-mails inviting me as an author to submit a manuscript to some plastic surgery journal or another. This is partially because the widespread adoption of open-access journals has made it possible for journal publishing to be a profitable business. In my opinion, this situation strongly resembles the Warring States period in ancient China, during which a unified kingdom developed from the chaos of multiple small states. Each journal introduces itself as a distinguished and promising journal; however, as is well known, the number of renowned journals in the plastic surgery field is very limited.

The prestigious journals in plastic surgery are usually published in Europe and North America, the birthplace of modern plastic surgery. After World Wars I and II, many soldiers were wounded and their appearance was significantly affected. There was a tremendous need for surgical procedures to correct aspects of the human form. Plastic surgery is one of the most recent specialties in the entirety of medicine, and it developed very rapidly based on its specific historical background. It is undeniable that doctors from Europe and North America played key roles in its development as a field.

Nonetheless, just think of the full scope of the peoples of the world. There are so many ethnicities outside of Europe and North America, all with different facial characteristics, body shapes, and cultures. Their needs for plastic surgery can likewise be somewhat different-that is, specific principles and procedures may be optimal for certain populations. Plastic surgery is different from other fields in medicine because it deals with human appearances. Some principles are the same across all popu- lations, but others can be different, because ethnicities differ in terms of their appearances and medical needs.

A representative example of this issue is aesthetic facial plastic surgery in Asians, many of whom do not have double folds in their eyelids; furthermore, Asians usually have a low projected nose and prominent zygomas. Plastic surgeons in this area make every effort to change patients' appearances to match their desired outcomes, and have-after decades of effort—succeeded in developing reliable methods of doing so. However, these techniques are somewhat meaningless for Caucasians and have not received much attention from researchers in North America and Europe.

Archives of Plastic Surgery hopes to bridge this gap. We want to reflect not only mainstream voices, but also those of othersof course, with a strong scientific basis. We pursue a balanced approach according to the needs of patients from diverse backgrounds. To achieve this goal, all manuscripts are reviewed by peers from throughout the world, including a statistician. We also provide free English proofreading and illustration services [1]. Archives of Plastic Surgery welcomes all manuscripts with new and innovative content. Of course, we also adhere to all ethical principles of medical publishing, because we at Archives of Plastic Surgery believe that ethics and scientific rigor are fundamental elements of medical research.

Through these efforts, we would like to share academic achievements in the field of plastic surgery with clinicians and patients from all over the world. Moreover, this journey is already in progress. 


\section{NOTES}

\section{Conflict of interest}

No potential conflict of interest relevant to this article was reported.

\section{ORCID}

Seung Yong Song

https://orcid.org/0000-0002-3145-7463

\section{REFERENCE}

1. Kim YH. The current position of Archives of Plastic Surgery and its future. Arch Plast Surg 2015;42:387-90.

Correspondence: Seung Yong Song

Department of Plastic and Reconstructive Surgery, Yonsei University College of Medicine

50-1 Yonsei-ro, Seodaemun-gu, Seoul 03722, Korea

Tel: +82-2-2228-2214, Fax: +82-2-393-6747,E-mail: iceberg554@naver.com

Received: November 4, $2020 \bullet$ Revised: November 8, $2020 \bullet$ Accepted: November 9, 2020

pISSN: 2234-6163 • elSSN: 2234-6171

https://doi.org/10.5999/aps.2020.02131 • Arch Plast Surg 2020;47:493-494 